\title{
Decentralized Congestion Management for Multilateral Transactions Based on Optimal Resource Allocation
}

\author{
Kai Liu, Yixin Ni, Senior Member, IEEE, Felix F. Wu, Fellow, IEEE, and T. S. Bi, Member, IEEE
}

\begin{abstract}
This paper proposes a decentralized model for dc load flow based congestion management for the forward markets via Optimal Resource Allocation (ORA). The available thermal capacities of possible congested transmission lines are considered as commonly shared resources for all bilateral and multilateral transactions in the market. In our model, each transaction maximizes its profit under the limits of transmission line capacities allocated by the ISO. The ISO searches the optimal allocation of line capacities to each transaction. Finally, the same market efficiency (social welfare) as centralized optimization can be reached. The ORAbased decentralized approach is more suitable for power market environment, since it always keeps the intermediate solution feasible during the iterations and does not require each transaction to submit their private and sensitive information. The mathematical model, computation procedure and relevant proof are presented. The computer test results from the IEEE 30 bus system illustrate the effectiveness of proposed approach.
\end{abstract}

Index Terms-Congestion management, decentralized optimization, optimal resource allocation, power market.

\section{INTRODUCTION}

$\mathbf{E}$ LECTRICITY markets have been developing rapidly in many parts of the world. However, the design of perfect power markets is still under investigation for various reasons. One of the most significant features of electricity market is that the commodity of electricity should be transferred through the network and the transmission line capacity limits should be considered at all times. The complicated issues of congestion management arise, which is relevant to both system operation security and market economic efficiency [1].

Mathematically, congestion management problem can be generally described as a centralized optimal power flow (COPF) problem with the objective function of maximizing social welfare and the constraints of load flow equations and operation

Manuscript received November 29, 2006; revised March 23, 2007. This work was supported in part by the National Key Basic Research Special Fund of China under Grant 2004CB217900, in part by Natural Science Foundation of China (50337010), and in part by the Open Foundation of the Key Laboratory of Power System Protection and Dynamic Security Monitoring and Control (North China Electric Power University) of the Ministry of Education under Grant KW02003. Paper no. TPWRS-00844-2006.

K. Liu, Y. Ni, and F. F. Wu are with the Department of Electrical and Electronic Engineering, The University of Hong Kong, HK SAR, China (e-mail: kailiu@eee.hku.hk; yxni@eee.hku.hk; ffwu@eee.hku.hk).

T. S. Bi is with the Key Laboratory of Power System Protection and Dynamic Security Monitoring and Control, Ministry of Education, North China Electric Power University, Beijing, China.

Color versions of one or more of the figures in this paper are available online at http://ieeexplore.ieee.org.

Digital Object Identifier 10.1109/TPWRS.2007.907540 limitations. However, the COPF approach has some apparent drawbacks in market environment. First, COPF requires the submission of detailed private information of market participants to the ISO that may include their cost/benefit functions. In the market environment, such sensitive information is a commercial secret that market participants are unwilling to disclose to the ISO. Secondly, the COPF by the ISO will be a "black box" that lacks transparency to market participants, since the congestion price is more likely "set" by the ISO, but not "discovered" through market mechanisms. As a centralized authority, the ISO has "super power," which is inconsistent with the principles of competitive markets. Moreover, for the congestion management of inter-regional trades, which can share the common resources (transmission lines, generation units, etc.) across regions efficiently and increase the scale of economy (e.g., in [2] and [3]), the decentralized optimal power flow (DOPF) approach is becoming more attractive for its possibility of using one agent for coordination and even without the existence of a real ISO for the entire system. Based on the above discussion, it is desirable to develop tools to address the issues of decentralized congestion management without sacrificing market efficiency.

In the study of decentralized congestion management, the decomposition techniques of a global OPF, which solve the problem iteratively by necessary information exchange and coordination strategies, are very attractive. In [2], the coordinated multilateral trade is proposed which separates the economy and reliability issues of system operation. It proves that the coordination result can achieve the maximal social welfare. In [5], the brokers of forward market arrange their contracts under the price signals of the congested paths updated by the public coordinator. This coordination process can converge to the optimal solution of original COPF. In [6], the Lagrange Relaxation (LR) decomposition technique is applied with the coordination of power exchanges of regional markets, and three algorithms for updating the Lagrange multipliers are compared. In [7] and [8], the concept of "dummy bus" is applied to separate the decoupled constraints by auxiliary-problem principle (APP) in [9], and hence to decompose global OPF by areas. The distributed optimization software in [10] based on the APP technique can calculate distributed optimal power flow with arbitrary division of the system. The decentralized dc load flow problem is converted to an optimization problem in [11], which targets at minimizing the difference between the variables of the decoupled constraints on borders and is solved by the APP technique. The calculation of decentralized power transfer distribution factors (PTDFs) and available transfer 
capacity (ATC) are also proposed. Until now, the most conventional decentralized approaches can be viewed as price-based methods. The coordinated signals are primarily relevant to Lagrange multiplier, the shadow price of congested paths in the congestion management problem. During the iterations, if any Lagrange multiplier is less than the real value in optimal solution, the infeasible intermediate solution appears and it is desired to be avoided.

This paper proposes the model of decentralized congestion management based on the new direction of the Optimal Resource Allocation (ORA) [12] of the transmission line capacity. The relevant concept was once applied to power markets in [13]. In this paper, we provide a more complete ORA-based model with relevant proof and propose two algorithms to solve the problem for the decentralized congestion management of forward markets.

The paper is organized as follows. In Section II, the mathematical model of COPF for the congestion management is converted to the decentralized model based on the ORA problem for transmission line capacity. The two suggested algorithms are presented in Section III. In Section IV, the test results on IEEE 30 bus system clearly show that the suggested approaches are effective. The proof for equivalence of proposed ORA-based decentralized model and COPF model is given in the Appendix.

\section{MATHEMATICAL FORMULATION}

\section{A. Outline of COPF Model for Congestion Management in Forward Markets}

We mainly focus on forward contract market for real power, where dc load flow is popular to use. The PTDFs are available to the market participants (transactions). In the perfect competitive market, the ISO adjusts the contracts to maximize the social welfare to achieve efficient operation with all constraints satisfied. This COPF is given by

$$
\begin{aligned}
& \max _{\mathbf{x}, \mathbf{y}} \sum_{k \in T} \sum_{j \in D^{(k)}} B_{j}^{(k)}\left(y_{j}^{(k)}\right)-\sum_{k \in T} \sum_{i \in G^{(k)}} C_{i}^{(k)}\left(x_{i}^{(k)}\right) \\
& \text { s.t. : } \quad \sum_{j \in D^{(k)}} y_{j}^{(k)}-\sum_{i \in G^{(k)}} x_{i}^{(k)}=0 \quad(k \in T) \\
& x_{i, \min }^{(k)} \leq x_{i}^{(k)} \leq x_{i, \max }^{(k)}\left(i \in G^{(k)}, k \in T\right) \\
& y_{j, \min }^{(k)} \leq y_{j}^{(k)} \leq y_{j, \max }^{(k)}\left(j \in D^{(k)}, k \in T\right) \\
& \sum_{k \in T} g_{k}^{(m)}\left(\mathbf{x}_{k}, \mathbf{y}_{k}\right) \leq P_{\max }^{(m)}(m \in I)
\end{aligned}
$$

where

$$
\begin{array}{ll}
T & \text { set of transactions in the market } \\
& T=\{1,2, \ldots, K\} ; \\
k & \text { index of each transaction, for all } k \in T ; \\
I & \text { set of transmission lines involved in congestion } \\
& \text { management } I=\{1,2, \ldots, M\} ; \\
m & \text { index of transmission lines involved in congestion } \\
& \text { management, for all } m \in I ;
\end{array}
$$

$G^{(k)} \quad$ set of generators in transaction $k$, for all $k \in T$;

$D^{(k)} \quad$ set of consumers in transaction $k$, for all $k \in T$;

$x_{i}^{(k)} \quad$ output of generator $i$ of transaction $k$, also an element of generator output vector $\mathbf{x}_{k}$ of transaction $k$, for all $i \in G^{(k)}$ and $k \in T$;

$y_{j}^{(k)} \quad$ demand of consumer $j$ of transaction $k$, an element of demand vector $\mathbf{y}_{k}$ of transaction $k$, for all $j \in D^{(k)}$ and $k \in T$;

$C_{i}^{(k)} \quad$ cost function of generator $i$ of transaction $k$, for all $i \in G^{(k)}$ and $k \in T$;

$B_{j}^{(k)} \quad$ benefit function of consumer $j$ of transaction $k$, for all $j \in D^{(k)}$ and $k \in T$;

$g_{k}^{(m)} \quad$ Load flow caused by transaction $k$ on line $m$, in which PTDFs are used, for all $k \in T$ and $m \in I$;

$P_{\max }^{(m)} \quad$ transfer limit of line $m$ in MW, for all $m \in I$.

The objective in (1) is to maximize total social welfare. The equality constraints in (2) denote the power balance for each transaction. The inequality constraints in (3) [or (4)] denote the output (or input) limits of generators (or consumers). Inequality constraints in (5) denote the line capacity limits. For simplicity the transmission lines is supposed to congest only in one direction. However, there is no difficulty to consider the limits for both directions by adding the opposite direction line capacity constraints into (5).

\section{B. Mathematical Model for ORA-Based Decentralized Approach}

The COPF model (1)-(5) is converted into ORA-based DOPF model below. Firstly we rewrite (1)-(5) to a simpler form. The decision variables of contract $k$ are defined as

$$
\mathbf{u}_{k}^{T} \stackrel{\text { def. }}{=}\left(\mathbf{x}_{k}^{T}, \mathbf{y}_{k}^{T}\right)(k \in T) .
$$

The social welfare related to transaction $k$ is rewritten as

$$
\begin{aligned}
W_{k}\left(\mathbf{u}_{k}\right) \stackrel{\text { def. }}{=} \sum_{j \in D^{(k)}} B_{j}^{(k)}\left(y_{j}^{(k)}\right) & \\
& -\sum_{i \in G^{(k)}} C_{i}^{(k)}\left(x_{i}^{(k)}\right) \quad(k \in T) .
\end{aligned}
$$

Equations (2)-(4) are local constraints of individual contracts that are not interactive among transactions. For transaction $k$, its local constraints are denoted as the feasible domain $\operatorname{Dom}_{k}$. The original COPF model is written to a simpler form as

$$
\begin{aligned}
\max & \sum_{k \in T} W_{k}\left(\mathbf{u}_{k}\right) \\
\text { s.t. }: & \sum_{k \in T} g_{k}^{(m)}\left(\mathbf{u}_{k}\right) \leqslant P_{\max }^{(m)} \quad(m \in I) \\
& - \text { global constraints } \\
\mathbf{u}_{k} & \in \operatorname{Dom}_{k} \quad(k \in T)-\text { local constraints. }
\end{aligned}
$$

In the ORA-based method, the ISO tries to optimally allocate the capacity resource of $M$ lines to $K$ transactions. We denote that the ISO allocates the capacity of line $m$ to transaction $k$ 
by the amount of $P_{k, \max }^{(m)}$, and define the Resource Allocation Weighing Matrix (RAWM) as $\boldsymbol{\alpha}$, an $M \times K$ matrix with its $m$ th row and $k$ th column element $\alpha_{k}^{(m)}$ denoting the proportion of the capacity of line $m$ allocated to the transaction $k$, then

$$
\begin{aligned}
P_{k, \max }^{(m)} & =P_{\max }^{(m)} \alpha_{k}^{(m)} \quad(m \in I, k \in T) \\
\sum_{k \in T} \alpha_{k}^{(m)} & =1 \quad(m \in I) .
\end{aligned}
$$

It is noticed that the index $\alpha_{k}^{(m)}$ in (8) can be negative caused by counter flow. By introducing $\alpha$, the COPF problem in (6) can be rewritten as

$$
\begin{aligned}
& \max \sum_{k \in T} W_{k}\left(\mathbf{u}_{k}\right) \\
& \text { s.t. }: g_{k}^{(m)}\left(\mathbf{u}_{k}\right) \leqslant P_{\max }^{(m)} \alpha_{k}^{(m)} \quad(k \in T, m \in I) \\
& \sum_{k \in T} \alpha_{k}^{(m)}=1 \quad(m \in I) \\
& \mathbf{u}_{k} \in \operatorname{Dom}_{k} \quad(k \in T) .
\end{aligned}
$$

The problem in (9) can be decomposed into $K$ independent sub-OPF problems corresponding to $K$ transactions under the fixed $\boldsymbol{\alpha}$ assigned by ISO. Thus, (9) is redefined as the optimization problem with decision variables of $\boldsymbol{\alpha}$ and the objective $\theta(\boldsymbol{\alpha})$ is

$$
\begin{aligned}
& \theta(\boldsymbol{\alpha})={ }^{\text {def. }} \\
& \max _{\mathbf{u}}\left\{\begin{array}{l|l}
\sum_{k \in T} W_{k}\left(\mathbf{u}_{k}\right) & \begin{array}{l}
g_{k}^{(m)}\left(\mathbf{u}_{k}\right) \leq P_{\max }^{(m)} \alpha_{k}^{(m)}(k \in T, m \in I) \\
\mathbf{u}_{k} \in \operatorname{Dom}_{k}(k \in T)
\end{array}
\end{array}\right\}
\end{aligned}
$$

which is equivalent to

$$
\begin{aligned}
& \theta(\boldsymbol{\alpha})=\sum_{k=1}^{K} \theta_{k}(\boldsymbol{\alpha}) \\
& \theta_{k}(\boldsymbol{\alpha}) \stackrel{\text { def. }}{=} \\
& \max _{\mathbf{u}_{k}}\left\{W_{k}\left(\mathbf{u}_{k}\right) \mid \begin{array}{l}
g_{k}^{(m)}\left(\mathbf{u}_{k}\right) \leqslant P_{\max }^{(m)} \alpha_{k}^{(m)} \\
\mathbf{u}_{k} \in \operatorname{Dom}_{k}
\end{array} \quad(m \in I)\right\} .
\end{aligned}
$$

By (8), the feasible domain $A$ of $\boldsymbol{\alpha}$ is

$$
A: \boldsymbol{\alpha} \in A=\left\{\boldsymbol{\alpha} \mid \sum_{k \in T} \alpha_{k}^{(m)}=1 \quad(m \in I)\right\} .
$$

The feasible domain $A$ can be further decomposed into $M$ independent subdomains $A^{(m)}$ based on (8), and we have

$$
\begin{aligned}
A & =A^{(1)} \times \cdots A^{(m)} \times \cdots A^{(M)} \\
A^{(m)}: \boldsymbol{\alpha}^{(m)} \in A^{(m)} & =\left\{\boldsymbol{\alpha}^{(m)} \mid \sum_{k \in T} \alpha_{k}^{(m)}=1\right\} .
\end{aligned}
$$

Based on (6) and (11), the master $\alpha$ - opt. problem for ISO based on ORA model should be

$$
\max _{\boldsymbol{\alpha}}\left\{\theta(\boldsymbol{\alpha})=\sum_{k \in T} \theta_{k}(\boldsymbol{\alpha}) \mid \boldsymbol{\alpha} \in A\right\}
$$

To solve the ORA problem for ISO in (14), we try to derive its optimality condition. From (8), the subdomain $A^{(m)}$ is a hyperplane. According to Karush Kuhn Tucker (KKT) necessary conditions, the optimal solution of (14) satisfies

$$
\frac{\partial(\theta(\boldsymbol{\alpha}))}{\partial \alpha_{1}^{(m)}}=\cdots \frac{\partial(\theta(\boldsymbol{\alpha}))}{\partial \alpha_{k}^{(m)}}=\cdots=\frac{\partial(\theta(\boldsymbol{\alpha}))}{\partial \alpha_{K}^{(m)}}(m \in I) .
$$

The explicit expression of $\theta(\boldsymbol{\alpha})$ is very difficult to derive. However, we can derive its first-order derivative (the sensitive information) $\partial(\theta(\boldsymbol{\alpha})) / \partial \alpha_{k}^{(m)}$ can be derived according to [15]

$$
\begin{aligned}
& \frac{\partial(\theta(\boldsymbol{\alpha}))}{\partial \alpha_{k}^{(m)}}=\frac{\partial\left(\theta_{k}(\boldsymbol{\alpha})\right)}{\partial \alpha_{k}^{(m)}} \\
& \frac{\partial \theta_{k}(\boldsymbol{\alpha})}{\partial P_{k, \max }^{(m)}}=\lambda_{k}^{(m)}, \quad \frac{\partial \theta_{k}(\boldsymbol{\alpha})}{\partial \alpha_{k}^{(m)}}=P_{\max }^{(m)} \lambda_{k}^{(m)} \\
& \quad(k \in T, m \in I) .
\end{aligned}
$$

In (16), $\lambda_{k}^{(m)}$ is the corresponding Lagrange multiplier of line $m$ limits in the sub-OPF problem of transaction $k$ in (11). The strict mathematical derivation is clearly given in [14, Ch. 12]. Understood from economic theory, (16) holds intuitively since Lagrange multipliers (shadow prices) denote the change rate of objective with respect to the constrained limited resource in optimization problem. If $\lambda_{k}^{(m)}$ is zero, the corresponding transfer limits is inactive. Meanwhile, adding a tiny capacity resource of line $m$ cannot cause any increment of profit for transaction $k$ (also for the social welfare); the change is zero. Thus, formula (16) holds according to whether or not $\lambda_{k}^{(m)}$ is zero. And the gradient matrix $\nabla \theta(\boldsymbol{\alpha})$ can be written as

$$
\begin{aligned}
\nabla \theta(\boldsymbol{\alpha})= & {\left[\begin{array}{ccc}
\frac{\partial \theta(\boldsymbol{\alpha})}{\partial \alpha_{1}^{(1)}} & \cdots & \frac{\partial \theta(\boldsymbol{\alpha})}{\partial \alpha_{K}^{(1)}} \\
\vdots & \ddots & \vdots \\
\frac{\partial \theta(\boldsymbol{\alpha})}{\partial \alpha_{1}^{(M)}} & \cdots & \frac{\partial \theta(\boldsymbol{\alpha})}{\partial \alpha_{K}^{(M)}}
\end{array}\right]=\left[\begin{array}{c}
\left(\frac{\partial \theta(\boldsymbol{\alpha})}{\partial \boldsymbol{\alpha}^{(1)}}\right)^{T} \\
\vdots \\
\left(\frac{\partial \theta(\boldsymbol{\alpha})}{\partial \boldsymbol{\alpha}^{(M)}}\right)^{T}
\end{array}\right] } \\
= & {\left[\begin{array}{ccc}
\lambda_{1}^{(1)} P_{\max }^{(1)} & \cdots & \lambda_{K}^{(1)} P_{\max }^{(1)} \\
\vdots & \ddots & \vdots \\
\lambda_{1}^{(M)} P_{\max }^{(M)} & \cdots & \lambda_{K}^{(M)} P_{\max }^{(M)}
\end{array}\right] } \\
= & {\left[P_{\max }^{(1)} \cdots P_{\max }^{(M)}\right]\left[\lambda_{k}^{(m)}\right]_{M \times K} . }
\end{aligned}
$$

Equation (15) becomes

$$
\lambda_{1}^{(m)}=\cdots=\lambda_{k}^{(m)}=\cdots=\lambda_{K}^{(m)} \quad(m \in I) .
$$

Equations (18) and (8) are the optimality necessary condition of (6) for ISO. The optimality necessary condition of master $\boldsymbol{\alpha}$ - opt. problem by ISO and the KKT necessary conditions of transactions are equivalent to the KKT necessary conditions of COPF. The proof is given in the Appendix. Generally, from the economic theory, if maximal social welfare is reached and no further resource reallocation is necessary, each line has the same economic efficiency for all transactions.

Equations (11), (12), and (14) form the ORA-based congestion management model, with its optimality condition for ISO in (18) and (6). The master $\boldsymbol{\alpha}$ - opt. problem by ISO and slave (sub) $\mathbf{u}_{k}$-opt. problem by each transaction will be solved iteratively to search the right $\alpha^{*}$ yielding $\mathbf{u}_{k}(k \in T)$ in transactions 


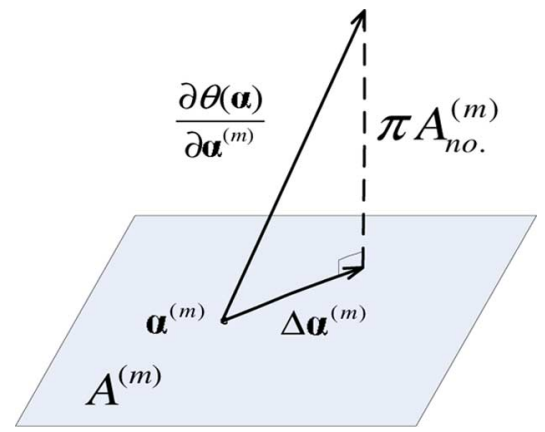

Fig. 1. Relation of $\partial \theta(\boldsymbol{\alpha}) / \partial \boldsymbol{\alpha}^{(m)}, \Delta \boldsymbol{\alpha}^{(m)}$ and $A^{(m)}$.

equal to the solution of COPF. The detailed solution method and procedure will be given in next section.

\section{RAWM UPDATING ALGORITHM FOR $\boldsymbol{\alpha}$ - OPT. AND Overall SOLUTION PROCEDURE}

\section{A. Gradient-Projection Method (GP)}

In the master $\boldsymbol{\alpha}$ - opt. problem by ISO, the explicit expression of $\theta(\boldsymbol{\alpha})$ in (10) cannot be attained and only the sensitive information matrix $\nabla \theta(\boldsymbol{\alpha})$ and feasible domain $A$ are available. The gradient projection approach will be proposed to solve such problems. The idea of the gradient-projection method is to project $\nabla \theta(\boldsymbol{\alpha})$ to its feasible domain $A$ to get feasible $\boldsymbol{\alpha}$ - opt. search direction $\Delta \boldsymbol{\alpha}$ to realize $\theta(\boldsymbol{\alpha}) \rightarrow$ max. During each iteration, $\nabla \theta(\boldsymbol{\alpha})$ is calculated according to (16) with $\left[\lambda_{k}^{* m)}\right]_{M \times K}$ submitted from each transaction $k \in T$, after its $\mathbf{u}_{k}$ - opt. problem (11) is solved $\Delta \boldsymbol{\alpha}$ is denoted as

$$
\Delta \boldsymbol{\alpha}^{T}=\left[\Delta \boldsymbol{\alpha}^{(1)}, \ldots, \Delta \boldsymbol{\alpha}^{(m)}, \ldots, \Delta \boldsymbol{\alpha}^{(M)}\right]
$$

where $\Delta \boldsymbol{\alpha}^{(m)} \in R^{K}(m \in I)$ is subsearch direction vector of line $m$. The relation of vectors $\partial \theta(\boldsymbol{\alpha}) / \partial \boldsymbol{\alpha}^{(m)}, \Delta \boldsymbol{\alpha}^{(m)}$ and domain $A^{(m)}$ is shown in Fig. 1, where $\Delta \boldsymbol{\alpha}^{(m)}$ is computed by the projecting $\partial \theta(\boldsymbol{\alpha}) / \partial \boldsymbol{\alpha}^{(m)}$ into hyperplane $A^{(m)}$.

The unit normal vector of $A^{(m)}$ is denoted as $A_{\text {no. }}^{(m)}$. The relation in Fig. 1 is given by ( $\pi$ is length coefficient)

$$
\begin{aligned}
& \frac{\partial \theta(\boldsymbol{\alpha})}{\partial \boldsymbol{\alpha}^{(m)}}-\Delta \boldsymbol{\alpha}^{(m)}=\pi A_{n o .}^{(m)} \\
& \left(\Delta \boldsymbol{\alpha}^{(m)}+\boldsymbol{\alpha}^{(m)}\right) \in A^{(m)}
\end{aligned}
$$

In the case of an optimal solution of (14), $\partial \theta(\boldsymbol{\alpha}) / \partial \boldsymbol{\alpha}^{(m)}$ parallels $A_{\text {no. }}^{(m)}$ (which is $\partial \theta(\boldsymbol{\alpha}) / \partial \boldsymbol{\alpha}^{(m)}=\pi A_{\text {no. }}^{(m)}$ ) and projection vector $\Delta \boldsymbol{\alpha}^{(m)}$ is zero for all $m \in I$. The "parallel" means that all elements of vector $\partial \theta(\boldsymbol{\alpha}) / \partial \boldsymbol{\alpha}^{(m)}$ are the same, since $A_{\text {no. }}^{(m)}$ is vector $[1]_{K \times 1}$ from (8) and (12). This analysis is consistent to optimality condition (15) and (18). To solve $\Delta \boldsymbol{\alpha}^{(m)}$, there are
$K$ equations from (19a) and one implicit $\left(\sum_{i=1}^{K} \Delta \alpha_{i}^{(m)}=0\right)$ from (19b) available and given by

$$
\begin{gathered}
{\left[\begin{array}{ccccc}
1 & 0 & \cdots & 0 & 1 \\
0 & 1 & & & \vdots \\
\vdots & & \ddots & & \vdots \\
0 & & & 1 & 1 \\
1 & \cdots & \cdots & 1 & 0
\end{array}\right] \times\left[\begin{array}{c}
\Delta \boldsymbol{\alpha}^{(m)} \\
\pi
\end{array}\right]} \\
=\left[\begin{array}{c}
\lambda_{1}^{(m)} \\
\vdots \\
\vdots \\
\lambda_{K}^{(m)}
\end{array}\right] P_{\max }^{(m)}, \quad(m \in I) .
\end{gathered}
$$

With (20), $\Delta \boldsymbol{\alpha}$ is attained from the independent calculation of $\Delta \boldsymbol{\alpha}^{(m)}(m \in I)$. The coefficient matrix in (20) is fixed and sparse, making the computation of (20) very fast. The RAWM $\boldsymbol{\alpha}$ can be updated in the direction of $\Delta \boldsymbol{\alpha}$ with suitable step-length $s$

$$
{ }^{\text {new }} \boldsymbol{\alpha}={ }^{\text {old }} \boldsymbol{\alpha}+s \boldsymbol{\Delta} \boldsymbol{\alpha} .
$$

The ${ }^{\text {new }} \boldsymbol{\alpha}$ will be forwarded to each transaction again to conduct the slave $\mathbf{u}_{k}$ - opt. problems and new $\left[\lambda_{k}^{* m}\right]_{M \times K}$ will be feedback to the ISO, forming one round of iteration. The coordination of master-slave optimization process will stop if the condition (18) can be satisfied. This gradient projection method has the first-order convergence quality.

\section{B. Trust Region and Quasi-Newton Method (TRQN)}

The well-known trust region and BFGS quasi-Newton (TRQN) optimization methods in 0 are implemented here as an alternative way to solve the problem at better convergence around the optimum, which is outlined below. For round $n$ with known ${ }^{n} \boldsymbol{\alpha}$, the trust-region problem for ISO in (14) is

$$
\begin{aligned}
\max _{\Delta \boldsymbol{\alpha}} \theta\left({ }^{n+1} \boldsymbol{\alpha}\right) & =\theta\left({ }^{n} \boldsymbol{\alpha}+\Delta \boldsymbol{\alpha}\right) \\
& =\nabla \theta\left({ }^{n} \boldsymbol{\alpha}\right) \Delta \boldsymbol{\alpha}+0.5(\Delta \boldsymbol{\alpha})^{\mathbf{T}} \nabla^{2} \theta\left({ }^{n} \boldsymbol{\alpha}\right) \Delta \boldsymbol{\alpha} \\
\text { s.t. : }{ }^{n+1} \boldsymbol{\alpha}= & \Delta \boldsymbol{\alpha}+{ }^{n} \boldsymbol{\alpha} \in A \\
& \|\Delta \boldsymbol{\alpha}\| \leq r
\end{aligned}
$$

where the objective is the local quadratic approximation of $\theta\left({ }^{n+1} \boldsymbol{\alpha}\right)$. The optimum of $\theta\left({ }^{n+1} \boldsymbol{\alpha}\right)$ is searched in the region with radius $r$. The local quadratic model requires the Hessian $\nabla^{2} \theta(\boldsymbol{\alpha})$. Since the exact Hessian is unknown, a positive definite approximation of $\nabla^{2} \theta(\boldsymbol{\alpha})$ is computed by the BFGS quasi-Newton algorithm as follows:

$$
\begin{aligned}
s_{n} & ={ }^{n} \boldsymbol{\alpha}-{ }^{n-1} \boldsymbol{\alpha} \\
y_{n}= & \nabla \theta\left({ }^{n} \boldsymbol{\alpha}\right)-\nabla \theta\left({ }^{n-1} \boldsymbol{\alpha}\right) \\
\nabla^{2} \theta\left({ }^{n+1} \boldsymbol{\alpha}\right)= & \nabla^{2} \theta\left({ }^{n} \boldsymbol{\alpha}\right) \\
& -\frac{\nabla^{2} \theta\left({ }^{n} \boldsymbol{\alpha}\right) s_{n} s_{n}^{T} \nabla^{2} \theta\left({ }^{n} \boldsymbol{\alpha}\right)}{s_{n}^{T} \nabla^{2} \theta\left({ }^{n} \boldsymbol{\alpha}\right) s_{n}}+\frac{y_{n} y_{n}^{T}}{y_{n}^{T} s_{n}}
\end{aligned}
$$




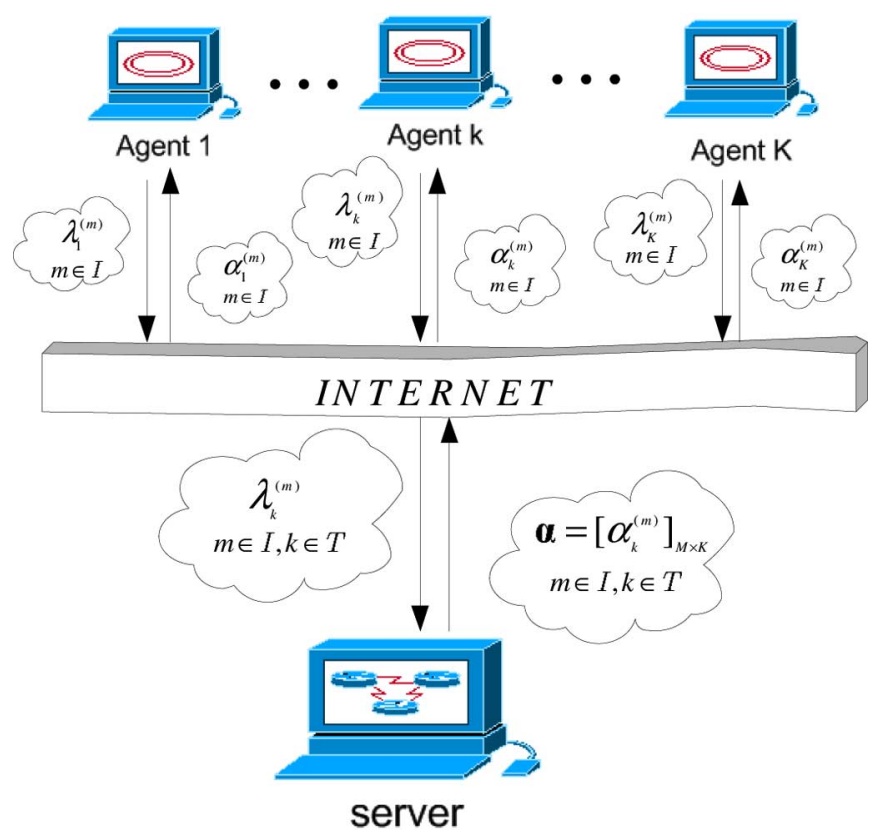

Fig. 2. Infrastructure of ORA-based decentralized congestion management by multi-agent system.

The second-order Hessian in (23) is based on gradient and Hessian information in the previous iterations. It provides more information of the problem to improve the convergence rate. The computation burden of (22) in the master $\boldsymbol{\alpha}$ - opt. problem by ISO is greater than (20). However, it takes less iteration to converge. We will discuss this point in next section.

\section{Scheme of Decentralized Congestion Management}

Based on the mathematical model and proposed RAWM updating algorithms, the general scheme for ORA-based decentralized congestion management for forward market is as follows.

Step 0: The ISO determines the possible congested transmission lines and give the initial RWAM $\boldsymbol{\alpha}$. If inexperienced, the ISO can uniformly assign line capacity to each transaction, i.e., $\alpha_{k}^{(m)}=1 / K(m \in I)$.

Step 1: ISO sends resource allocation signal $\alpha_{k}^{(m)}(m \in$ $I, k \in T$ ) to the corresponding transaction.

Step 2: Each transaction solves $\mathbf{u}_{k}$ for (11) to pursue the maximal profit under limits $\alpha_{k}^{(m)}(m \in I)$ allocated by ISO, and then sends its $\lambda_{k}^{(m)}(m \in I)$ back to the ISO.

Step 3: The ISO check whether the received Lagrange multipliers from each transaction can be close enough for all lines according to (18). If not, ISO updates $\boldsymbol{\alpha}$ by GP method in (20) or TRQN method in (22) and (23), and go to step 2.

Nowadays, the implementation of coordinated algorithm suggested above is possible with the fast development of information technology, such as World-Wide-Web and multi-agent technology in 0. A schematic diagram (see Fig. 2) shows the brief implementation of the algorithm through a multi-agent system (MAS). In the MAS, agent 1 to $K$ will solve (11) on transactions participants' behalves, while the server will solve the ISO resource allocation problem with necessary information exchanged over the Internet.

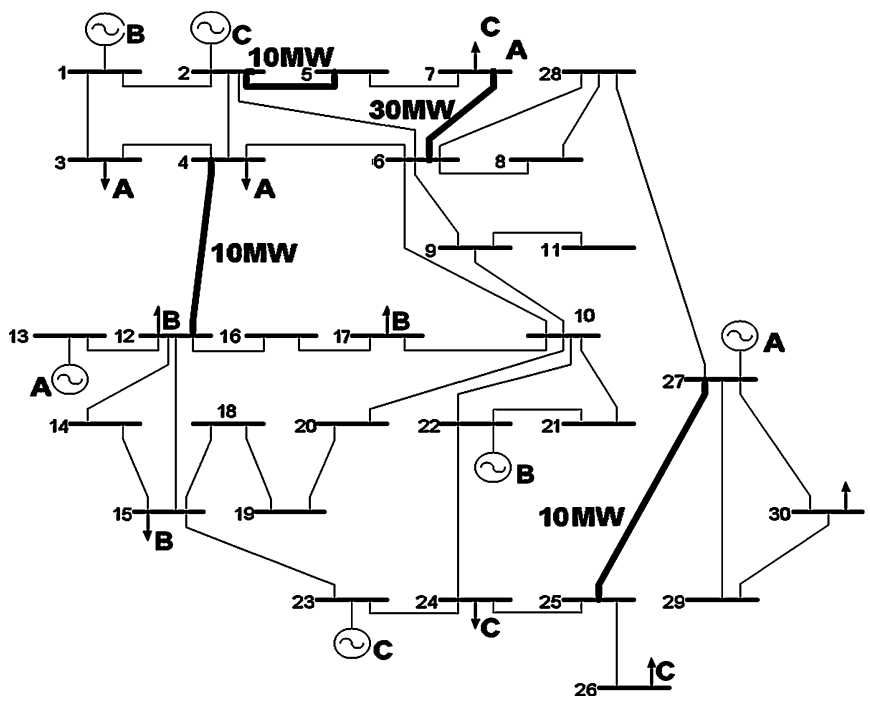

Fig. 3. IEEE 30-bus system.

TABLE I

Cost AND BENEFIT FunCTION OF TEST CASE

\begin{tabular}{ccccc}
\hline \hline Gen. bus & $\begin{array}{l}\text { Marginal cost } \\
(10 \$ / \mathrm{MWh})\end{array}$ & Con. Bus & $\begin{array}{c}\text { Marginal benefit } \\
(10 \$ / \mathrm{MWh})\end{array}$ \\
\hline & 13 & $2+0.02 \mathrm{q}$ & 3 & $3.88-0.03 \mathrm{q}$ \\
$\mathrm{A}$ & 27 & $1.75+0.0175 \mathrm{q}$ & 4 & $3.8-0.05 \mathrm{q}$ \\
& 1 & $3+0.025 \mathrm{q}$ & 12 & $3.8-0.02 \mathrm{q}$ \\
\hline & 22 & $3+0.025 \mathrm{q}$ & 15 & $3.8-0.04 \mathrm{q}$ \\
$\mathrm{B}$ & 23 & $2.8+0.0625 \mathrm{q}$ & 24 & $3.95-0.02 \mathrm{q}$ \\
\hline & 2 & $2.3+0.0283 \mathrm{q}$ & 26 & $3.7-0.02 \mathrm{q}$ \\
\hline
\end{tabular}

TABLE II RESULTS OF COPF

\begin{tabular}{cccccccc}
\hline \hline & & Max & A & B & C & $\begin{array}{c}\text { Sum } \\
\text {. }\end{array}$ & $\begin{array}{c}\text { Lagrange } \\
\text { multiplier }\end{array}$ \\
\hline $\begin{array}{c}\text { Capacity } \\
\text { used }\end{array}$ & Line 2-5 & 10 & 2.35 & 1.85 & 5.80 & 10.00 & 1.68 \\
$(\mathrm{MW})$ & Line 6-7 & 30 & 19.00 & -1.85 & 8.78 & 25.93 & 0 \\
& Line 4-12 & 10 & 26.82 & -15.38 & -1.44 & 10.00 & 0.82 \\
& Line 25-27 & 10 & 7.84 & 0.75 & 1.41 & 10.00 & 2.07 \\
\hline \multicolumn{2}{l}{ Trans. profit (M\$) } & & 86.15 & 15.70 & 21.05 & 122.90 & \\
\hline \hline
\end{tabular}

*Only lines 2-5, 4-12 and 25-27 are congested, and line 6-7 is not congested.

\section{TEST REsult}

The IEEE 30-bus test system (see Fig. 3) is used for testing, which is conducted by one computer with series computation. We consider the case that there are three multilateral transactions (A, B, C) and each has five participants of two generators and three consumers with their marginal cost function of generators and marginal benefit function of consumers listed in Table I. In total, four transmission lines are involved in the congestion management and shown as bold lines in Fig. 2.

Table II shows the results of COPF with negative values for counter flow. Lines 2-5, 4-12, and 25-27 are congested and the corresponding Lagrange Multipliers are greater than 0 , and Line 4-12 will be further analyzed to show the validity of the suggested methods. 


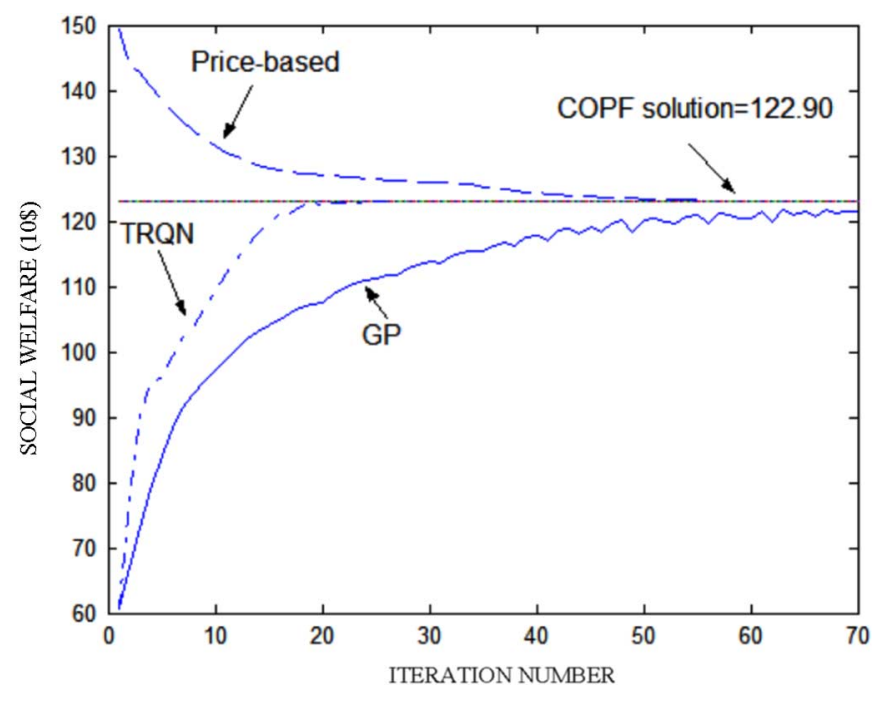

Fig. 4. Social welfare during the iterations.

TABLE III

COMPARISON OF CPU TIME BY GP AND TRQN METHODS

\begin{tabular}{cccc}
\hline \hline $\begin{array}{c}\text { Methods } \\
\text { by ISO }\end{array}$ & $\begin{array}{c}\text { Total CPU time } \\
\text { (series compute) }\end{array}$ & $\begin{array}{c}\text { Average ISO } \\
\text { CPU time. }\end{array}$ & $\begin{array}{c}\text { Optimal obj. value } \\
\text { (\%COPF[122.90]) }\end{array}$ \\
\hline GP $(\mathrm{n}=25)$ & $4.82 \mathrm{~s}$ & $10^{-6} \mathrm{~s}$ & $121.68(99.01 \%)$ \\
TRQN $(\mathrm{n}=70)$ & $3.76 \mathrm{~s}$ & $0.1086 \mathrm{~s}$ & $122.89(99.99 \%)$ \\
\hline \hline
\end{tabular}

In Fig. 4, the dynamic process of social welfare during iteration by different decentralized approaches is compared with the COPF solution. The price-based method 0,0 converges to the solution of COPF from the infeasible domain, since it underestimates the initial congested price. The two suggested ORA-based methods reach the COPF solution effectively with their intermediate solutions keeping feasible. Thus, the ORA-based method has the advantage over price-based method for that it can stop at any iteration with a feasible overall solution.

Table III compares the two suggested $\boldsymbol{\alpha}$-opt. methods. Since series computation is used, there is much room for reducing the total CPU time when parallel computation is used for individual transaction. In Table III, it can be seen that TRQN method takes fewer rounds and lower overall CPU time to converge to the optimal solution than GP method does, but at the cost of longer CPU time of ISO.

Fig. 5(a) shows the dynamic process of allocated capacities of congested line 4-12 to transactions A, B, C by the TRQN method. The allocated capacity of line 4-12 for transaction A is increasing; while for transactions $\mathrm{B}$ and $\mathrm{C}$, it decreases and converges to negative value as counter flow. Jointly, three transactions make full and optimal utilization of the capacity of line 4-12 to yield maximal social welfare. Fig. 5(b) shows dynamic process of Lagrange multipliers of transactions A, B, C for line 4-12. They tend to be identical at the final optimization solution and satisfy (18). Shown in Fig. 4, both allocated capacities and Lagrange multipliers tend to converge to that in COPF results.

\section{CONCLUSION}

A novel ORA-based decentralized approach is proposed for congestion management in the electricity forward market. The capacity of possible congested transmission lines is optimally

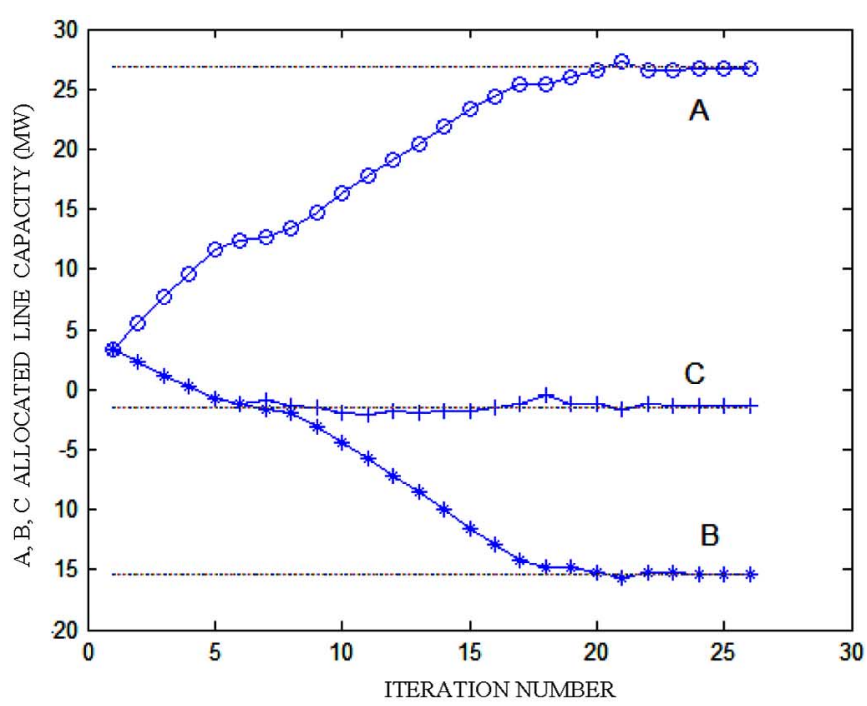

(a)

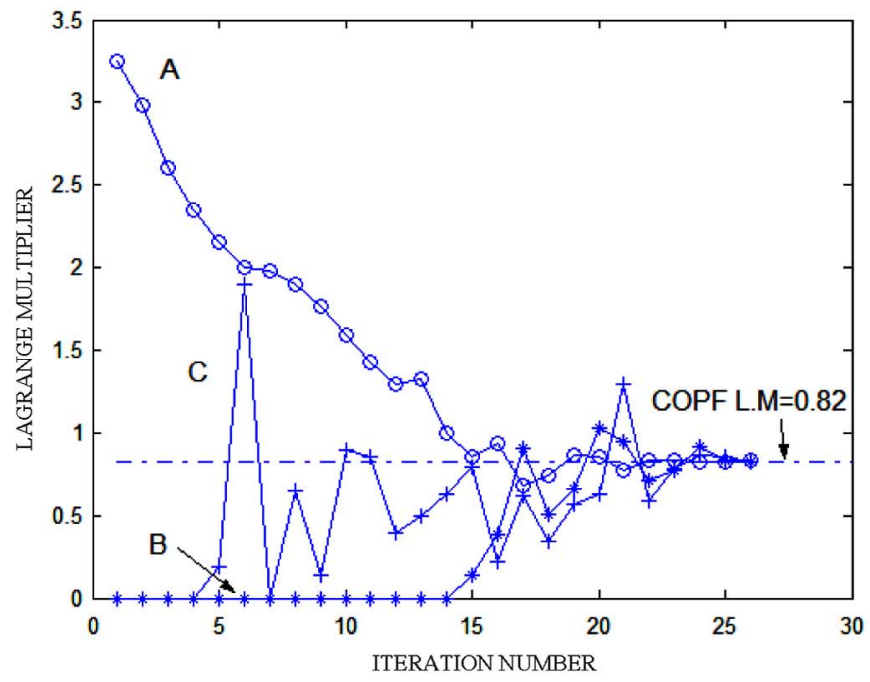

(b)

Fig. 5. (a) Allocated capacities and (b) Lagrange multipliers of line 4-12.

allocated to individual transactions for maximizing the social welfare. The derivation of mathematical model and relevant proof show the equivalence of the proposed decentralized model with COPF. Testing on the IEEE 30-bus system shows the proposed model and solution algorithms are effective. The distinct feature of ORA-based method is that it can always keep the intermediate solution feasible during iterations. To use the decentralized model, the impacts on limited resources by individuals are required to be able to rewrite in the form of (5). The ORA decentralized model is promising to apply to some other issues of electricity market. Further research work will be conducted to extend the method to ac load flow based on OPF with reactive power and voltage level impacts included.

\section{APPENDIX}

The Appendix gives the proof for the equivalence of the KKT condition of COPF and ORA-based decentralized model.

The local constraints $\mathbf{u}_{k} \in \operatorname{Dom}_{k}(k \in T)$ are generally described as $d_{l}^{(k)}\left(\mathbf{u}_{k}\right) \leqslant L_{l}^{(k)}\left(l \in V_{k}, k \in T\right)$ where $V_{k}$ is the set 
of constraints equations in $\mathbf{u}_{k} \in \mathrm{Dom}_{k}$. The KKT conditions of two models are shown in $\mathrm{P} 1$ and $\mathrm{P} 2$, respectively.

P1: The KKT condition of COPF in (6).

Lagrange function of (6) is

$$
\begin{aligned}
& L(\mathbf{u}, \lambda, \beta) \\
& =\sum_{k \in T} W_{k}\left(\mathbf{u}_{k}\right)+\sum_{m \in I} \lambda^{(m)}\left(P_{\max }^{(m)}-\sum_{k \in T} g_{k}^{(m)}\left(\mathbf{u}_{k}\right)\right) \\
& \quad+\sum_{k \in T} \sum_{l \in V_{k}} \beta_{l}^{(k)}\left(L_{l}^{(k)}-d_{l}^{(k)}\left(\mathbf{u}_{k}\right)\right) .
\end{aligned}
$$

The partial derivative equations of the Lagrange function are

$$
\begin{aligned}
\frac{\partial L}{\partial \mathbf{u}}= & \left(\frac{\partial L}{\partial \mathbf{u}_{1}} ; \cdots \frac{\partial L}{\partial \mathbf{u}_{k}} \cdots ; \frac{\partial L}{\partial \mathbf{u}_{K}}\right)=\mathbf{0} \quad(k \in T) \\
\frac{\partial L}{\partial \mathbf{u}_{k}}= & \frac{\partial\left(\sum_{k \in T} W_{k}\left(\mathbf{u}_{k}\right)\right)}{\partial \mathbf{u}_{k}} \\
& -\frac{\partial\left(\sum_{m \in I} \lambda^{(m)} \sum_{k \in T} g_{k}^{(m)}\left(\mathbf{u}_{k}\right)\right)}{\partial \mathbf{u}_{k}} \\
& -\frac{\partial\left(\sum_{k \in T} \sum_{l \in V_{k}}\left(\beta_{l}^{(k)} d_{l}^{(k)}\left(\mathbf{u}_{k}\right)\right)\right)}{\partial \mathbf{u}_{k}} \\
= & \frac{\partial W_{k}\left(\mathbf{u}_{k}\right)}{\partial \mathbf{u}_{k}}-\sum_{m \in I} \lambda^{(m)} \frac{\partial g_{k}^{(m)}\left(\mathbf{u}_{k}\right)}{\partial \mathbf{u}_{k}} \\
& -\sum_{l \in V_{k}} \beta_{l}^{(k)} \frac{\partial d_{l}^{(k)}\left(\mathbf{u}_{k}\right)}{\partial \mathbf{u}_{k}} .
\end{aligned}
$$

Suppose that $\mathbf{u}_{k}(k \in T)$ is the solution of (6). Then there are Lagrange Multipliers $\lambda^{(m)}$ for line transfer limits and $\beta_{l}^{(k)}$ for local constraints $\left(m \in I ; k \in T ; l \in V_{k}\right)$, satisfying the KKT conditions given by

$$
\begin{aligned}
& \frac{\partial W_{k}\left(\mathbf{u}_{k}\right)}{\partial \mathbf{u}_{k}}-\sum_{m \in I} \lambda^{(m)} \frac{\partial g_{k}^{(m)}\left(\mathbf{u}_{k}\right)}{\partial \mathbf{u}_{k}} \\
& -\sum_{l \in V_{k}} \beta_{l}^{(k)} \frac{\partial d_{l}^{(k)}\left(\mathbf{u}_{k}\right)}{\partial \mathbf{u}_{k}}=\mathbf{0} \quad(k \in T) \\
& P_{\max }^{(m)}-\sum_{k \in T} g_{k}^{(m)}\left(\mathbf{u}_{k}\right) \geq 0 \quad(m \in I) \\
& \lambda^{(m)}\left(P_{\max }^{(m)}-\sum_{k \in T} g_{k}^{(m)}\left(\mathbf{u}_{k}\right)\right)=0 \quad(m \in I) \\
& \lambda^{(m)} \geq 0 \quad(m \in I) \\
& d_{l}^{(k)}\left(\mathbf{u}_{k}\right) \leq L_{l}^{(k)} \quad\left(l \in V_{k}, k \in T\right) \\
& \beta_{l}^{(k)}\left(L_{l}^{(k)}-d_{l}^{(k)}\left(\mathbf{u}_{k}\right)\right)=0 \quad\left(l \in V_{k}, k \in T\right) \\
& \beta_{l}^{(k)} \geq 0 \quad\left(l \in V_{k}, k \in T\right) .
\end{aligned}
$$

P2: The KKT conditions for transactions and ISO of DOPF supposing that $\mathbf{u}_{k}(k \in T)$ is the solution of (11) for each transaction, there are $\lambda_{k}^{(m)}, \alpha_{k}^{(m)}, \beta_{l}^{(k)}\left(m \in I ; k \in T ; l \in V_{k}\right)$ sat- isfying the KKT conditions of transaction given by (f-4)-(f-6) and optimality condition of ISO given by (f-7)

$$
\begin{aligned}
& \frac{\partial W_{k}\left(\mathbf{u}_{k}\right)}{\partial \mathbf{u}_{k}}-\sum_{m \in I} \lambda_{k}^{(m)} \frac{\partial g_{k}^{(m)}\left(\mathbf{u}_{k}\right)}{\partial \mathbf{u}_{k}} \\
& \quad-\sum_{l \in V_{k}} \beta_{l}^{(k)} \frac{\partial d_{l}^{(k)}\left(\mathbf{u}_{k}\right)}{\partial \mathbf{u}_{k}}=\mathbf{0} \quad(k \in T) \\
& P_{\max }^{(m)} \alpha_{k}^{(m)}-g_{k}^{(m)}\left(\mathbf{u}_{k}\right) \geq 0 \quad(m \in I, k \in T) \\
& \lambda_{k}^{(m)}\left(P_{\max }^{(m)} \alpha_{k}^{(m)}-g_{k}^{(m)}\left(\mathbf{u}_{k}\right)\right)=0 \quad(m \in I, k \in T) \\
& \lambda_{k}^{(m)} \geq 0 \quad(m \in I, k \in T) \\
& d_{l}^{(k)}\left(\mathbf{u}_{k}\right) \leq L_{l}^{(k)} \quad\left(l \in V_{k}, k \in T\right. \\
& \beta_{l}^{(k)}\left(L_{l}^{(k)}-d_{l}^{(k)}\left(\mathbf{u}_{k}\right)\right)=0 \quad\left(l \in V_{k}, k \in T\right) \\
& \beta_{l}^{(k)} \geq 0 \quad\left(l \in V_{k}, k \in T\right. \\
& \lambda_{1}^{(m)}=\cdots=\lambda_{k}^{(m)}=\cdots=\lambda_{K}^{(m)} \quad(m \in I) \\
& \sum_{k \in T} \alpha_{k}^{(m)}=1 \quad(m \in I) .
\end{aligned}
$$

The procedure to prove $\mathrm{P} 1 \Leftrightarrow \mathrm{P} 2$ is: if $\mathrm{P} 1$ holds, according to $\mathrm{P} 1$, we can find $\lambda_{k}^{(m)}, \alpha_{k}^{(m)}, \beta_{l}^{(k)}\left(m \in I, k \in T, l \in V_{k}\right)$ satisfying equations for $\mathrm{P} 2$ from (f-4) to (f-7) with $\mathbf{u}_{k}(k \in T)$ unchanged as in $\mathrm{P} 1(\mathrm{P} 1 \Rightarrow \mathrm{P} 2)$; and vice versa $(\mathrm{P} 2 \Rightarrow \mathrm{P} 1)$. (Here, uniqueness of the Lagrange multipliers is not required.) Proof of $\mathrm{P} 1 \Rightarrow \mathrm{P} 2$ : We can set $\alpha_{k}^{(m)}$ satisfying (f-7-2) as

$$
\alpha_{k}^{(m)}= \begin{cases}g_{k}^{(m)}\left(\mathbf{u}_{k}\right) / P_{\max }^{(m)}, & \text { for } k \neq K \quad(m \in I) \\ 1-\sum_{k=1}^{K-1} \alpha_{k}^{(m)} & \text { for } k=K .\end{cases}
$$

By setting $\lambda_{k}^{(m)}=\lambda^{(m)}$, (f-7-1) and (f-5-3) holds. Also, fixing $\mathbf{u}_{k}$ and $\beta_{l}^{(k)}$ unchanged in $P 1$, (f-4) and (f-6) holds.

When $k \neq K$ then

$$
P_{\max }^{(m)} \alpha_{k}^{(m)}-g_{k}^{(m)}\left(\mathbf{u}_{k}\right)=0
$$

when $k=K$, by (f-2-1) then

$$
\begin{aligned}
P_{\max }^{(m)}\left(1-\sum_{k=1}^{K-1} \alpha_{k}^{(m)}\right)-g_{K}^{(m)} & \left(\lambda_{k}^{(m)}\right) \\
& =P_{\max }^{(m)}-\sum_{k=1}^{K} g_{k}^{(m)}\left(\mathbf{u}_{k}\right) \geqslant 0 .
\end{aligned}
$$

Therefore, (f-5-1) holds. When $\lambda^{(m)}=0$, (f-5-2) holds. When $\lambda^{(m)} \neq 0$, if $k \neq K$ then

$$
\lambda_{k}^{(m)}\left(P^{(m)} \alpha_{k}^{(m)}-g_{k}^{(m)}\left(\mathbf{u}_{k}\right)\right)=0
$$

and if $k=K$, then by (f-2-2) there is

$$
\begin{aligned}
\lambda_{k}^{(m)}\left(P_{\max }^{(m)} \alpha_{k}^{(m)}-g_{k}^{(m)}\left(\mathbf{u}_{k}\right)\right) & \\
= & \lambda_{k}^{(m)}\left(P_{\max }^{(m)}-\sum_{k=1}^{K} g_{k}^{(m)}\left(\mathbf{u}_{k}\right)\right)=0 .
\end{aligned}
$$

Therefore, (f-5-2) still holds. 
Proof $P 2 \Rightarrow P 1$ : By setting $\lambda^{(m)}=\lambda_{k}^{(m)}$ corresponding to (f-7-1), fixing $\mathbf{u}_{k}$ and $\beta_{l}^{(k)}$ unchanged in $P 2$, (f-5-3), (f-3) and (f-1) hold.

By (f-7) and (f-5-1), then

$$
\begin{aligned}
& P_{\max }^{(m)}-\sum_{k=1}^{K} g_{k}^{(m)}\left(\mathbf{u}_{k}\right) \\
& =P_{\max }^{(m)} \sum_{k=1}^{K} \alpha_{k}^{m}-\sum_{k=1}^{K} g_{k}^{(m)}\left(\mathbf{u}_{k}\right) \\
& =\sum_{k=1}^{K}\left(P_{\max }^{(m)} \alpha_{k}^{m}-g_{k}^{(m)}\left(\mathbf{u}_{k}\right)\right) \geq 0 .
\end{aligned}
$$

Therefore, (f-2-1) holds. By (f-7) and (f-5-2), it has

$$
\begin{aligned}
\lambda^{(m)} & \left(P_{\max }^{(m)}-\sum_{k=1}^{K} g_{k}^{(m)}\left(\mathbf{u}_{k}\right)\right) \\
= & \lambda^{(m)}\left(\sum_{k=1}^{K} P_{\max }^{(m)} \alpha_{k}^{(m)}-\sum_{k=1}^{K} g_{k}^{(m)}\left(\mathbf{u}_{k}\right)\right) \\
= & \sum_{k=1}^{K} \lambda_{k}^{(m)}\left(P_{\max }^{(m)} \alpha_{k}^{(m)}-g_{k}^{(m)}\left(\mathbf{u}_{k}\right)\right)=0 .
\end{aligned}
$$

Therefore, (f-2-2) holds.

Thus from the proof presented above, P1 and P2 are equivalent. The KKT conditions of the COPF and ORA-based DOPF model are consistent to each other.

It should be noticed that in some special cases when a local limit and a global limit become active at the same point for transaction $k$, the relevant $\lambda_{k}^{(m)}$ may sharply change (or say, jump) from one value denoted as $-\lambda_{k}^{(m)+}$ to the other value denoted as $\lambda_{k}^{(m)-} q$

- right at that point. Actually in this situation, $\lambda_{k}^{(m)}$ has multiple solutions which vary from $\lambda_{k}^{(m)+}$ to $\lambda_{k}^{(m)-}{ }^{-}$satisfying the KKT conditions. According to the above explanation, the equivalence of KKT conditions of COPF and DOPF models still holds, and, for this special case, the ISO in DOPF model can still select proper $\lambda_{k}^{(m)} \in\left[\lambda_{k}^{(m)-}, \lambda_{k}^{(m)+}\right]$ to satisfy (18). But computer program should be developed properly to handle such a special case.

\section{REFERENCES}

[1] R. D. Christie, B. F. Wollenberg, and I. Wangenstee, "Transmission management in the deregulated environment," Proc. IEEE, vol. 88, no. 2, pp. 170-195, Feb. 2000.

[2] European Transmission System Operator (ETSO). [Online]. Available: http://www.eTSO-net.org/.

[3] PJM-MISO Joint and Common Market White Paper. [Online]. Available: http://www.joint-andcommon.com/index.html.

[4] F. F. Wu and P. Varaiya, "Coordinated multilateral trades for electric power networks: Theory and implementation," Int. J. Elect. Power Energy Syst., vol. 21, no. 2, pp. 75-102, 1999.
[5] P. Wei, Y. Ni, and F. F. Wu, "Decentralized approach for congestion management and congestion price discovering," Proc. Inst. Elect. Eng., vol. 149, no. 6, pp. 645-652, 2002.

[6] J. A. Aguado and V. H. Quintana, "Inter-utilities power-exchange coordination: A market-oriented approach," IEEE Trans. Power Syst., vol. 16, no. 3, pp. 513-519, Aug. 2001.

[7] B. H. Kim and R. Baldick, "Coarse-grained distributed optimal power flow,” IEEE Trans. Power Syst., vol. 12, no. 2, pp. 932-939, May 1997.

[8] R. Baldick, "A fast distributed implementation of optimal power flow," IEEE Trans. Power Syst., vol. 14, no. 3, pp. 858-864, Aug. 1999.

[9] G. Cohen, "Auxiliary problem principle and decomposition of optimization problems," J. Optim. Theory Applicat., vol. 32, no. 3, pp. 277-305, Nov. 1980.

[10] J. Contreras, "DistOpt: A software framework for modeling and evaluating optimization problem solutions in distributed environments," $J$. Parallel Distrib. Comput., vol. 60, pp. 741-763, 2000.

[11] A. G. Bakirtzis and P. N. Biskas, "Decentralised dc load flow and applications to transmission management," Proc. Inst. Elect. Eng., vol. 149, no. 5, pp. 600-606, 2002.

[12] J. Kornai and T. Liptpk, "Two level planning," Econometrica, vol. 33, no. 1, pp. 141-168, Jan. 1965.

[13] S. Hao, "Decentralized approach to intermarket congestion management," IEEE Trans. Power Syst., vol. 20, no. 2, pp. 675-683, May 2005.

[14] J. Nocedal and S. J. Wright, Numerical Optimization. New York: Springer, 1999

[15] P. Maes, Designing Autonomous Agents. Cambridge, MA: Elsevier/MIT Press, 1990.

[16] X. Wang, Y.H. Song, and Q. Lu, "Lagrange decomposition approach to active power congestion management across interconnected regions," Proc. Inst. Elect. Eng., vol. 148, no. 5, pp. 497-503, 2001.

Kai Liu was born in Beijing, China, in 1982. He received the B.Eng. degree in electrical engineering from Tsinghua University, Beijing, China, and is now pursuing the M.Phil. degree at the University of Hong Kong, Hong Kong, China.

His currently research topics include decentralized optimization and investment in power market.

Yixin Ni (SM'94) received the B.Eng., M.Eng., and Dr.Eng. degrees, all in electrical engineering, from Tsinghua University, Beijing, China.

She was Professor and Director of the National Power System Laboratory, Tsinghua University, and is now with the University of Hong Kong, Hong Kong, China. Her research interests are power system stability and control, FACTS, and electric energy industry restructuring.

Dr. Ni is an associate editor of the IEEE TRANSACTIONS ON POWER SYSTEMS.

Felix F. Wu (SM'86-F'89) received the Ph.D. degree from the University of California at Berkeley (UCB).

$\mathrm{He}$ is now a Chair Professor of electrical engineering and Vice President of the University of Hong Kong, Hong Kong, China. Prior to that, he was a Professor and Vice-Chair of Department of the Electrical Engineering and Computer Science Department, UCB. His research interests are electric energy industry restructuring, power system investment planning, design of modern control centers, distribution automation, and distributed processing.

T. S. Bi (M'02) received the Ph.D. degree in electrical engineering from the University of Hong Kong, Hong Kong, China, in 2002.

She is now a Professor at North China Electric Power University, Beijing, China. Her research interests are power system control, WAMS applications, and fault diagnosis. 